\title{
Erratum
}

\section{Cinchona Alkaloids in Asymmetric Organocatalysis}

Tommaso Marcelli, * Henk Hiemstra* Synthesis 2010, 1229.

In section 4.4 (p. 1255) references $212 \mathrm{a}$ and $212 \mathrm{~b}$ are erroneously mentioned as contributions from the same authors. In fact, they are independent publications from two different research groups. We apologize for the oversight. 